\title{
A case of coronavirus disease 2019 (COVID-19) presenting after coronary artery bypass grafting
}

\author{
Michael Salna, MD, Antonio Polanco, MD, Vinayak Bapat, MD, Isaac George, MD, \\ Michael Argenziano, MD, and Koji Takeda, MD, PhD, New York, NY
}

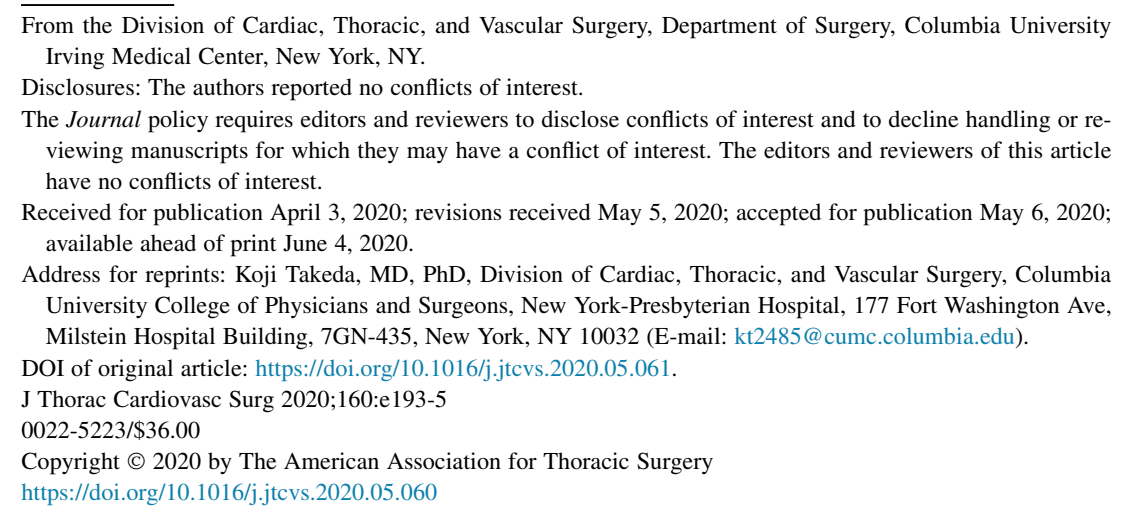

Coronavirus disease 2019 (COVID-19) is the clinical manifestation of infection with severe acute respiratory syndrome coronavirus-2 and most often presents with fevers and respiratory symptoms that can progress from simple pneumonia to severe acute respiratory distress syndrome (ARDS) and shock. ${ }^{1}$ As the number of cases surpasses 1 million worldwide, evidence is emerging that there are a variety of cardiovascular manifestations of the disease that can adversely affect outcomes. ${ }^{2}$

Herein, we present a case of a patient who underwent coronary artery bypass surgery and was found to be positive for COVID-19 with a course complicated by severe ARDS and probable myocarditis and microangiopathic coagulopathy with embolic phenomena.

\section{CASE REPORT}

The family provided informed consent for this case report. A 57-year-old man with hypertension, poorly controlled type 2 diabetes (A1c 12.2), previous STsegment elevation myocardial infarction in 2009, and a forced expiratory volume in 1 second/forced vital capacity of $83 \%$, who recently returned from Manilla and Hong Kong, presented with an non-ST-segment elevation myocardial infarction and was found to have severe multivessel coronary disease as well as a left ventricular ejection fraction of $35 \%$ with anteroseptal hypokinesis. He continued to have angina despite maximal medical therapy but notably did not have any cough, dyspnea, or anosmia. Due to lesions not amenable to percutaneous coronary interventions, he underwent an urgent 3-vessel coronary artery bypass surgery. Coming off bypass, an intraoperative

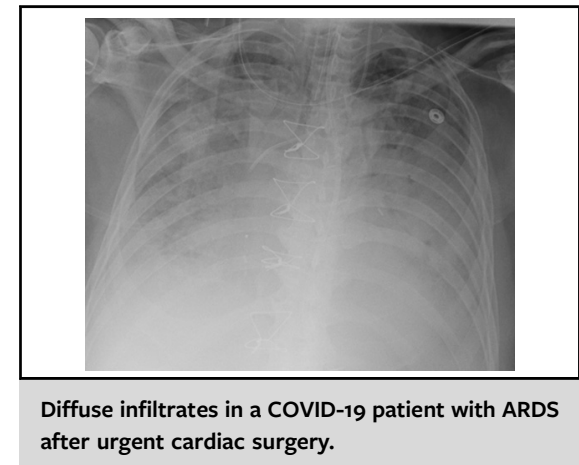

CENTRAL MESSAGE

COVID-19 has a variety of cardiovascular manifestations and may mimic postcardiotomy complications. For the near future, all patients undergoing urgent cardiac surgery should be tested for COVID-19.

See Commentaries on pages e197 and e198.

transesophageal echocardiogram demonstrated a left ventricular ejection fraction of $35 \%$ with global hypokinesis. $\mathrm{He}$ was transferred to the intensive care unit on low-dose pressors and an intra-aortic balloon pump. Total bypass time was 110 minutes, and crossclamp time was 93 minutes.

The following day, the patient became febrile to $38.2^{\circ} \mathrm{C}$, required increasing vasopressor support, and developed inferior and anterolateral ST changes on electrocardiogram (Figure 1). Given rising high-sensitivity troponins (max $8987 \mathrm{ng} / \mathrm{mL}$ ) and creatine kinase myocardial band levels $(217.7 \mathrm{ng} / \mathrm{mL}$ ), he was taken to the catheterization lab, where all grafts were found to be patent, with good runoff. The next morning, his right upper extremity, without previous intervention, was noted to be a cool, and an occlusive thrombus was found in the distal brachial artery. Several hours later, a stroke code was called for acute left gaze deviation despite being neurologically intact previously; a computed tomography of the head demonstrated 


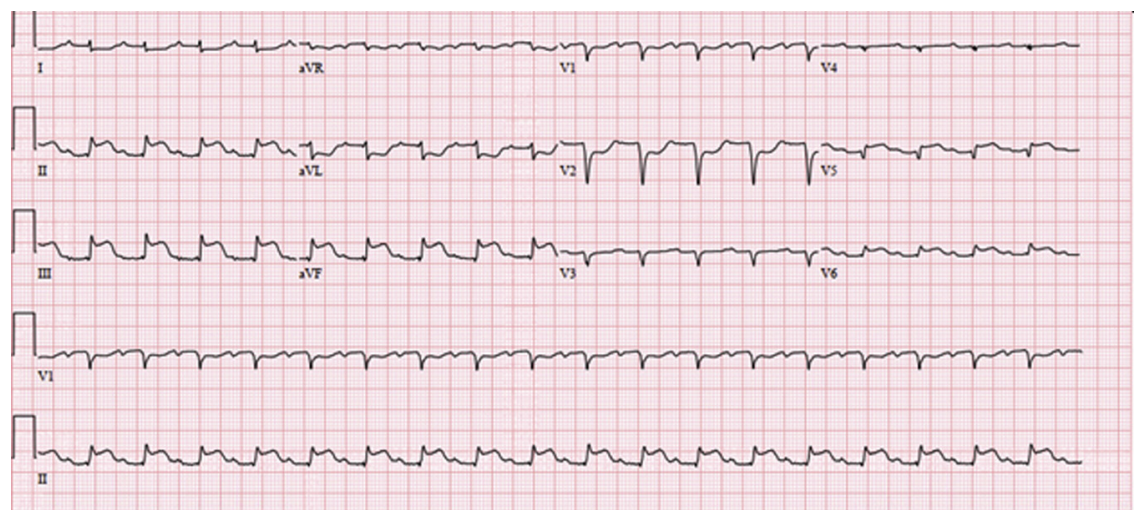

FIGURE 1. Electrocardiogram on postoperative day 1 demonstrating ST-segment elevations in the inferior leads and V5-V6 as well as ST-segment depressions in leads I, aVL, and V1-V2.

bilateral vertebral artery occlusions, a new right carotid occlusion, and parieto-occipital and basilar infarcts for which he received tissue plasminogen activator and a mechanical embolectomy with good flow post-recanalization.

The patient was vasoplegic through the fourth postoperative day and received 2 doses of $25 \mathrm{mg}$ intravenous hydrocortisone with mild improvement in vasopressor doses. However, the following night he again became febrile to $38.6^{\circ} \mathrm{C}$, his oxygenation progressively worsened with a arterial partial pressure of oxygen to fraction of inspired oxygen ratio $<100$ on assist volume-control ventilation 14/ $400 / 70 \% / 5$ (gas 7.29/49/38), and he developed worsening diffuse pulmonary infiltrates on chest radiograph (Figure 2). The ARDSnet protocol was initiated with initial settings of $16 / 380 / 100 \% / 18$. His severe acute respiratory syndrome coronavirus- 2 reverse transcriptase-polymerase chain reaction assay came back positive, and he was started on oral hydroxychloroquine and azithromycin.

Over the successive days, he suffered persistent mixed septic and cardiogenic shock in addition to ARDS. At the same time, his D-dimer climbed to $7.7 \mu \mathrm{g} / \mathrm{mL}$, fibrinogen to $670 \mathrm{mg} / \mathrm{dL}$, troponins remained consistently above $7000 \mathrm{ng} / \mathrm{L}$, and platelets fell to $6 \times 10^{3} / \mu \mathrm{L}$ despite transfusions. His partial thromboplastin times were consistently 40 to 60 seconds despite only receiving $5000 \mathrm{mg}$ of subcutaneous heparin twice daily with a heparin-activity-level of $<0.1$ $\mathrm{U} / \mathrm{mL}$ and a negative serotonin release assay to rule out heparin-induced-thrombocytopenia. His interleukin-6 levels were elevated at $18 \mathrm{pg} / \mathrm{mL}$ (normal level <5), although throughout his course he maintained only a mild leukocytosis of $9-11 \times 10^{3} \mathrm{U} / \mathrm{L}$. Given his nonrecovering neurologic status, inability to be weaned from pressors or the ventilator, care was withdrawn and the patient died.

\section{DISCUSSION}

Although the cardiovascular effects of COVID-19 are still being defined, this case illustrates several potential complications of the virus that may cloud clinical judgment in the setting of recent cardiac surgery. This patient's postoperative

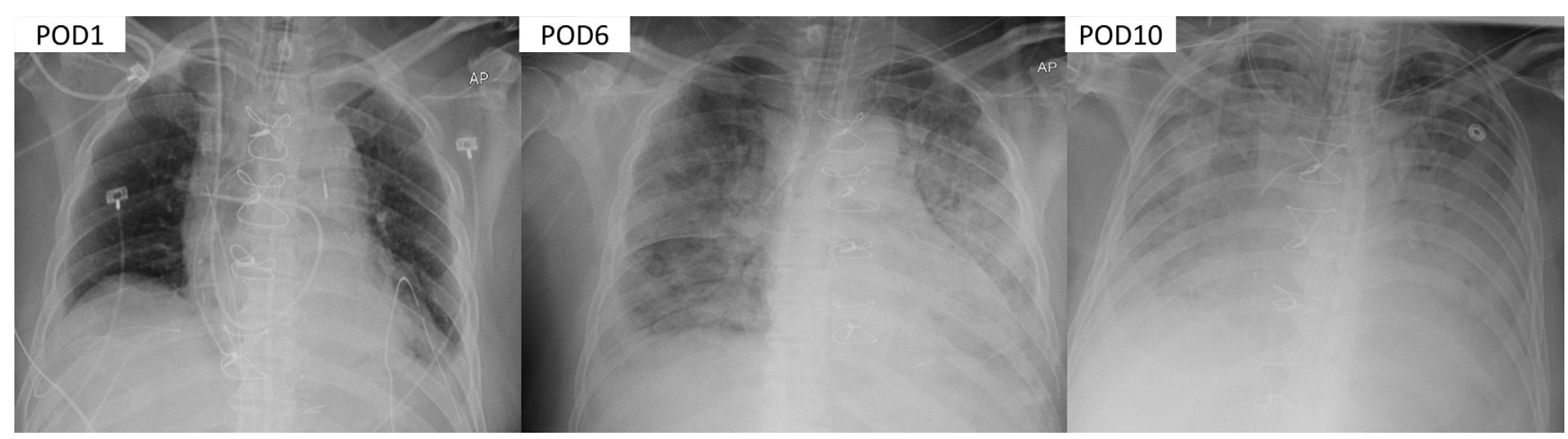

FIGURE 2. Progression of COVD-19 ARDS development in a patient after coronary artery bypass grafting. POD, Postoperative day. 
shock suggested ischemia from graft failure, although subsequent catheterization ruled this out. In hindsight, it is possible that the global hypokinesis seen on echocardiography was due to a combination of stunned myocardium and viral myocarditis, which became persistently worse over the coming days, eventually leading to refractory shock. Given the patient's poor neurologic status, potential stroke exacerbation with systemic heparinization, and profound thrombocytopenia, all in the context of resource scarcity during the COVID-19 pandemic, we decided not to pursue additional mechanical circulatory support, for which we are very strict with patients with COVID-19.

A second consideration is that of microangiopathic thrombosis, which may have also contributed to this patient's myocardial depression. While he maintained palpable pulses without evidence of distal ischemia, his rising D-dimer and fibrinogen levels coupled with profound thrombocytopenia, and possibly even his ischemic stroke, suggest some type of coagulopathy-laboratory trends that seem to be consistent with previous data. ${ }^{3,4}$ Anecdotal evidence of hypercoagulability in patients with COVID-19 has already begun appearing in the literature in the setting of unexplained pulmonary emboli. ${ }^{5}$

Emergent cardiac surgery cannot stop in the wake of a global pandemic. This case illustrates the potential cardiovascular manifestations of COVID-19 and its potential to mimic postcardiotomy complications. Our institution now routinely screens all patients undergoing surgery for COVID-19 and will defer nearly all cases in positive patients. This will likely continue for the near future, as the rapid deterioration of this patient suggests he contracted COVID-19 preoperatively without showing symptoms. In hindsight, had it been known this patient was positive, we would have recommended targeted percutaneous coronary interventions even if suboptimal. While these cases should be deferred, a deeper understanding of the cardiovascular complications of this virus after cardiac surgery can expedite diagnosis and initiation of appropriate treatment.

\section{References}

1. Bhatraju PK, Ghassemieh BJ, Nichols M, Kim R, Jerome KR, Nalla AK, et al. Covid-19 in critically ill patients in the Seattle region-case series. $N$ Engl J Med. 2020;382:2012-22

2. Bonow RO, Fonarow GC, O'Gara PT, Yancy CW. Association of coronavirus disease 2019 (COVID-19) with myocardial injury and mortality. JAMA Cardiol. March 27, 2020 [Epub ahead of print].

3. Chen N, Zhou M, Dong X, Qu J, Gong F, Han Y, et al. Epidemiological and clinical characteristics of 99 cases of 2019 novel coronavirus pneumonia in Wuhan, China: a descriptive study. Lancet. 2020;395:507-13.

4. Wang D, Hu B, Hu C, Zhu F, Liu X, Zhang J, et al. Clinical characteristics of 138 hospitalized patients with 2019 novel coronavirus-infected pneumonia in Wuhan, China. JAMA. 2020;323:1061-9.

5. Danzi GB, Loffi M, Galeazzi G, Gherbesi E. Acute pulmonary embolism and COVID-19 pneumonia: a random association? Eur Heart J. 2020;41: 1858 . 